\title{
Human-Robot Interaction Strategies for Unobtrusively Acquiring Health-Related Data
}

\author{
Maria Dagioglou and \\ Stasinos Konstantopoulos \\ Institute of Informatics and \\ Telecommunications, NCSR 'Demokritos' \\ Ag. Paraskevi, Greece \\ Email: \{mdagiogl,konstant\}@iit.demokritos.gr
}

\author{
A. Seza Doğruöz \\ Netherlands Institute for Advanced Studies \\ Wassenaar, Netherlands \\ Email: a.s.dogruoz@gmail.com
}

\author{
Franziska Kirstein \\ Univeristy of Southern Denmark \\ Odense, Denmark \\ Email: franziska.kirstein@gmail.com
}

\begin{abstract}
Using robotic home assistants as a platform for remote health monitoring offers several advantages, but also presents considerable challenges related to both the technical immaturity of home robotics and to user acceptance issues. In this paper we explore tablets and similar mobile devices as the medium of communication between robots and their users, presenting relevant current and planned research in human-robot interaction that can help the telehealth community circumvent technical shortcomings, improve user acceptance, and maximize the quality of the data collected by robotic home assistants.
\end{abstract}

Keywords-Human-Robot Interaction, Telehealth, HealthRelated Data

\section{INTRODUCTION}

Using robotic home assistants as a platform for remote health monitoring is a growing research area in healthcare at home. Robots' mobility, in particular, offers the advantage of being able to collect data from angles and ranges that cannot be easily obtained from alternative setups and, furthermore, robots can be used more pro-actively ensuring that data is collected regularly.

Health monitoring mostly targets elderly users. For this reason, the home assistant functionality should integrate HumanRobot Interaction (HRI) strategies and methods that enhance robot's acceptance by the users: the procedure for making service requests should be easy and natural, it should create opportunities to unobtrusively collect health monitoring data, and it should avoid making unrealistic demands on the robot's cognitive capabilities.

Using an application via a mobile phone or a tablet to interact with the robot has multiple advantages in this respect. To begin with, it is possible to develop applications adapted toward personal background (e.g. gender, age, culture) and cognitive and physical abilities (e.g. special apps for disabled users). Moreover, the design and implementation of an app is cost efficient. Besides from interacting with the robot, an application in combination with the sensors that are integrated (or can be integrated) in a mobile device can be used to monitor activities of daily life (ADL). This can be a valuable tool for both informal care-givers and medical professionals who can use data collected by such applications. In addition, an application on a mobile device that is not part of the 'robot body' allows multiple users to have access to the same robot.
In a scenario where a robot is used in a hospital one robot could be serving multiple users.

In the rest of the paper, we present the literature on the factors that influence the success of HRI (Section II), discuss the challenges and opportunities of using mobile devices to control robotic home assistants while remotely collecting health monitoring data (Section III), and conclude by presenting a research plan for developing the necessary technologies that will allow home healthcare community to successfully grasp the opportunity offered by the rapid advancement of mobile devices and home robotics (Section IV).

\section{BACKGROUND}

In the field of Human-Robot Interaction (HRI), there is a rich literature on how the effectiveness of interaction depends on factors related to user personality and background, as well as the characteristics of the robot, and the medium of the interaction.

From the perspective of the users, the range of factors that influence the acceptance of healthcare robots by elderly people has been found to be very wide: age, needs, gender, experience with technology (and robots in particular), cognitive ability and education, culture, role and anxiety and attitudes towards robots [3]. Several studies have mapped how user characteristics and background determine what robot behaviours are appropriate. Takayama and Pantofaru [27], for instance, determined that robot gaze has a different influence on women than men and that people who have experience with robots or have pets feel more comfortable when being approached by a robot on short distance. More recent research has demonstrated that social robots are more easily accepted when they conform with stereotypes that match their occupational role (e.g., healthcare, security) to the 'gender' and 'personality' that they are designed to possess [29].

A robot's embodiment plays a key role in its assistive effectiveness [28]. Research shows that users find an embodied robot more appealing than a virtual agent [3], [31]. It is important that the robot's embodiment fits its abilities and intelligence in order for the user not to get confused [19], [28]. If a robot looks simple, users do not expect it to perform on a high level. If it looks technically complex, user will expect the robot to perform at a high level. 
Different interfaces have been integrated in order to realize a non-expert's interaction with the robot. Most of these interfaces integrate human-human interaction features such as natural language, voice recognition, gaze and gestures [2], [8], [16]. Other assistive robots are controlled by the user with the help of handheld devices [7], [21], [25]. Panek and colleagues [17] use a LED projector unit on the back of an Aldebaran Nao robot to enhance assistance.

Using tablets as an HRI medium has been a natural development, as tablets are becoming familiar, in fact, ubiquitous devices. Assistant robots like the Care-O-Bot [5], Pearl [18], HOBBIT [4] are equipped with tablets that can be used to communicate with them. Closest to our setup is the assistive robot CASERO that can be controlled by a tablet to conduct simple carrying tasks [9]. Also, Granata and colleagues [6] explored the pictures that should be used when designing a tablet interface of the Kompai robot for people with cognitive disorders.

An application, especially when used by elderly citizens, needs to be user friendly. However, Salvini et al. [23] differentiate between usability and user's willingness to interact with the robot as two separate aspects of human robot interaction: If a robot is not accepted by elderly users, the user-friendliness of the design is useless. Another advantage of using an application is the ease of accessibility and use: end-users can download the application on their own, familiar, smart phones or tablets. Ease of accessibility can influence liking the system [32]. At the same time, the development and distribution of an application is easier and more cost-efficient.

\section{OPPORTUNITIES AND CHALLENGES}

The advantages of using robots in telehealth applications have been previously discussed in more detail [10]. This paper focuses on using tablets, smartphones, and similar mobile devices to communicate with a robot that is used as a telehealth data collection platform. One immediate advantage is that the data collected from the robot's sensors can be complemented by data collected form the mobile device's sensors. Collecting telehealth data from mobile devices, laptops, and desktop computers is well-studied and significant results are immediately applicable [1].

The further advantage and challenge is to design the robot/mobile system is such a way that they not only complement each other in data collection, but also that this complementarity stresses the unobtrusive and natural character of the system, so that the system as a whole is both more useful and more easily accepted than either the robot or the mobile device alone. Suppose that user communicates with a robot via an application to request a service, such as that the robot brings them something. There are multiple ways to set up the system, depending on what we want users to perceive as being their 'sentient' interaction peer:

- The robot presents itself as the cognitive system, with user and robot using the app as a communication channel.

- The robot presents itself as the cognitive system, and offers the app as a means of interaction.
- The app presents itself as the cognitive system. The user interacts with the app, and the app might decide to control the robot (or other devices) in order to achieve a goal.

Communication strategies such as direct or indirect speech, a robot's appearance and presence and interaction distance play a great role in HRI [26]. Salem et al. [22] found out that the interaction context has a greater influence on participant's perception of the HRI interaction than the use of verbal politeness strategies. Even though not all humanhuman interaction features can be applied to a human robot interaction, Torrey et al. [30] suggest that natural humanhuman assistive interactions can help to plan effective human robot assistants. Strait et al. [26] claim that results differ when communication strategies are tested via indirect interaction scenarios where the user watched the interaction passively such as in online questionnaires than when users actually experience a real interaction with a robot. There is need for more and in-depth investigation to understand how users communicate through apps with Robots.

The robot does not verbally speak to the user because when it uses the application to communicate. In addition to the application, the robot itself can move, make sounds and simple gestures such as shaking his head. There is research on robotic behaviour concerning the use of space [11], [14], [15], [27], gestures [13], and sounds [12], [20]. However, these factors have so far not been studied in combination with a mobile device.

We set-up a simple demonstration to gain some insights into the factors that influence the users perception and that can be manipulated by system design, as well as into the challenges entailed in interacting with a robot that carries out a daily life task. In this demonstration, the user uses a mobile device to order coffee, following on-screen instructions. The app and instructions are designed so that the user provides a closeup video during ordering. This video is used by the robot to identify and deliver the coffee to the right person (Fig. 1).

The mobile device app communicates with the user by the use of text combined with pictures. Text and picture are combined according to the principle of redundancy, repeating the same key idea in the text as well as in the picture [24]. Moreover, the robot produces 'beep' sounds to catch user's attention during critical moments of interaction, such as when the user has to place the cup on the robot's tray.

In the simple demonstration we set up, the participants $(n=5)$ had problems in conceiving the application and the robot as an integrated system and also they had trouble giving useful feedback given the set-up of the experiment. This raises the issue of how to set-up an HRI experiment in a more realistic background in order to be able to study the aforementioned perceptions of the app-robot integration.

\section{Concluding Remarks And Research Plan}

We have presented ideas and preliminary experiments on using an integrated robot/mobile application system as a data collection platform for telehealth applications. At the core of our work is the idea that user acceptance can be improved by combining immediate utility for the user with obtaining sensor data. 


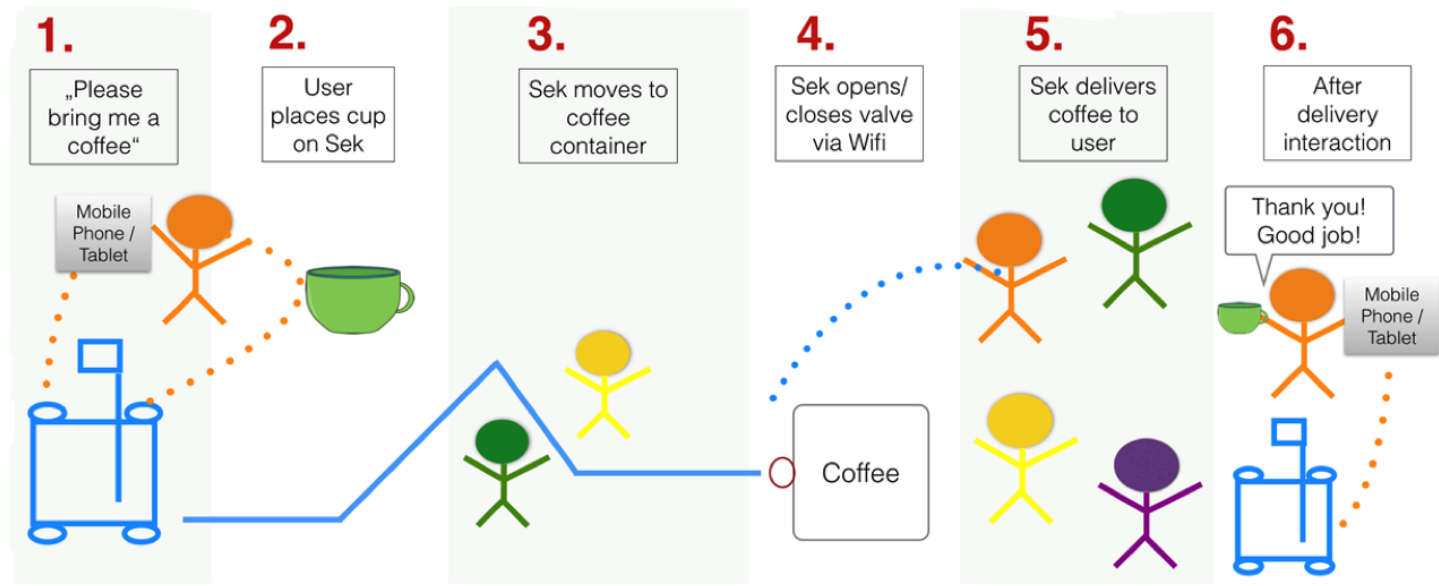

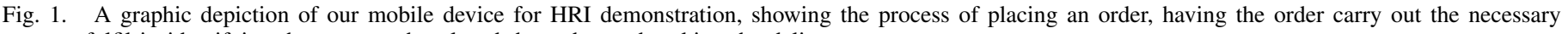
steps to fulfil it, identifying the person who placed the order, and making the delivery.

We are using RoboCoffee as a demo where the system has to be designed in such a way that it can collect the footage it needs in order to be able to identity the person who ordered the coffee without scaring 'customers' away into preferring the non-robotic alternative of getting coffee. The RoboCoffee demo has been developed on GitHub and is publicly available at https://github.com/roboskel/RoboCoffee

Our research plan involves investigating how humans communicate with the robot through an application. Having observed that most of the people that participated in the demonstration above did not feel that they interacted with the robot, we find it interesting to carry out further experiments where the robot and an individual entity is made explicit at different levels, exploring how people perceive the interaction with the system and how this perception influences what they expect from it. Put simply, we will explore if people have higher expectations from systems presenting themselves a robot that from systems presenting themselves as a mobile app, even when identical functionality is offered by both systems.

Another issue that we will explore further is how obtrusive do people consider providing a video to be. In our current setup, ensuring that the data provided by the user at ordering time is adequate for identifying the user at delivery time, we guided users by a bounding box in which they had to place their face to make sure that the right capture is achieved at ordering time. This can be avoided using face recognition to automatically place the the box on the image instead of asking the user to place their face so that it falls within a fixed box. It is not, however, necessarily the case that this is desired, as it can be construed as misleading the user about how the images captured from the mobile device are used. We plan to investigate user reactions to different positions along the axis of completely explicitly collecting telehealth related data to collecting all data without ever making the telehealth application explicit.

\section{ACKNOWLEDGEMENT}

The authors gratefully acknowledge the help of Andreas Lydakis, NCSR 'Demokritos', for assistance with his expertise in robot software engineering issues.
The work described here was partially carried out at the 2014 International Research-Centred Summer School (http://irss.iit.demokritos.gr) and in the context of Roboskel, the robotics activity of the Institute of Informatics and Telecommunications, NCSR 'Demokritos'. For more details please visit http://roboskel.iit.demokritos.gr

\section{REFERENCES}

[1] Alexander Artikis, Panagiotis D. Bamidis, Antonis Billis, Charalampos Bratsas, Christos Frantzidis, Vangelis Karkaletsis, Manousos Klados, Evdokimos Konstantinidis, Stasinos Konstantopoulos, Dimitris Kosmopoulos, Homer Papadopoulos, Stavros Perantonis, Sergios Petridis, and Constantine S. Spyropoulos. Supporting tele-health and AI-based clinical decision making with sensor data fusion and semantic interpretation: The USEFIL case study. In Proceedings of the Internation Workshop on Artificial Intelligence and NetMedicine (NetMed 2012), held at ECAI 2012. Montpellier, France, 27 August 2012, August 2012.

[2] R. Atienza and A. Zelinsky. Active gaze tracking for human-robot interaction. In Proceedings of the Fourth IEEE International Conference on Multimodal Interfaces, pages 261-266, 2002.

[3] E. Broadbent, R. Stafford, and B. MacDonald. Acceptance of healthcare robots for the older population: Review and future directions. International Journal of Social Robotics, 1(4):319-330, 2009.

[4] David Fischinger, Peter Einramhof, Walter Wohlkinger, K Papoutsakis, Peter Mayer, Paul Panek, T Koertner, S Hofmann, Antonis Argyros, Markus Vincze, et al. HOBBIT - the mutual care robot. In Proceedings of ASROB 2013, workshop held in conjunction with IEEE/RSJ IROS, Japan, 2013.

[5] B. Graf, U. Reiser, M. Hägele, K. Mauz, and P. Klein. Robotic home assistant care-o-bot $\AA ; 3$ - product vision and innovation platform. In IEEE Workshop Advanced on Robotics and its Social Impacts (ARSO), 2009, pages 139-144, Nov 2009.

[6] C. Granata, M. Chetouani, A Tapus, P. Bidaud, and V. Dupourque. Voice and graphical -based interfaces for interaction with a robot dedicated to elderly and people with cognitive disorders. In RO-MAN, 2010 IEEE, pages 785-790, Sept 2010.

[7] Cheng Guo and Ehud Sharlin. Exploring the use of tangible user interfaces for human-robot interaction: a comparative study. In In Proceedings of the SIGCHI Conference on Human Factors in Computing Systems, CHI '08, ACM, 2008.

[8] A. Haasch, S. Hohenner, S. Huwel, M. Kleinehagenbrock, S. Lang, I. Toptsis, G. A. Fink, J. Fritsch, B. Wrede, and G. Sagerer. Biron the bielefeld robot companion. In in Proc. Int. Workshop on Advances in Service Robotics, pages 27-32. Fraunhofer IRB Verlag, 2004.

[9] T. Jacobs and B. Graf. Practical evaluation of service robots for support and routine tasks in an elderly care facility. In Advanced Robotics and 
its Social Impacts (ARSO), 2012 IEEE Workshop on, pages 46-49, May 2012.

[10] Vangelis Karkaletsis and Stasinos Konstantopoulos. Embodiment and audio-visual perception in tele-health environments. In Proceedings of the 6th International Conference on Pervasive Technologies Related to Assistive Environments (PETRA 2013), Rhodes Island, Greece, 29-31 May 2013, 2013.

[11] Daphne Karreman, Lex Utama, Michiel Joosse, Manja Lohse, Betsy van Dijk, and Vanessa Evers. Robot etiquette: How to approach a pair of people? In Proceedings of the 2014 ACM/IEEE International Conference on Human-robot Interaction, HRI '14, pages 196-197, New York, NY, USA, 2014. ACM.

[12] Hideki Kozima, Marek Piotr Michalowski, and Cocoro Nakagawa Keepon: A playful robot for research, therapy, and entertainment. International Journal of Social Robotics, 1(1):3-18, January 2009.

[13] Ross Mead. Space, speech, and gesture in human-robot interaction. In Proceedings of the 14th ACM International Conference on Multimodal Interaction, ICMI '12, pages 333-336, New York, NY, USA, 2012. ACM.

[14] Ross Mead and Maja J. Mataric. Autonomous control of humanrobot spacing: A socially situated approach. In Proceedings of the 1st Symposium on Spatial User Interaction, SUI '13, pages 91-91, New York, NY, USA, 2013. ACM.

[15] J. Mumm and B. Mutlu. Human-robot proxemics: Physical and psychological distancing in human-robot interaction. In Human-Robot Interaction (HRI), 2011 6th ACM/IEEE International Conference on, pages 331-338, March 2011.

[16] Kai Nickel and Rainer Stiefelhagen. Real-time recognition of 3dpointing gestures for human-machine-interaction. In Bernd Michaelis and Gerald Krell, editors, Pattern Recognition, volume 2781 of Lecture Notes in Computer Science, pages 557-565. Springer Berlin Heidelberg, 2003.

[17] Paul Panek, Georg Edelmayer, Peter Mayer, Christian Beck, and Marjo Rauhala. User acceptance of a mobile led projector on a socially assistive robot. In Reiner Wichert and Birgid Eberhardt, editors, Ambient Assisted Living, Advanced Technologies and Societal Change, pages 77-91. Springer Berlin Heidelberg, 2012.

[18] M. Pollack, S. Engberg, J.T. Matthews, Sebastian Thrun, L. Brown, D. Colbry, C. Orosz, B. Peintner, S. Ramakrishnan, J. Dunbar-Jacob, C. McCarthy, Michael Montemerlo, Joelle Pineau, and Nicholas Roy. Pearl: A mobile robotic assistant for the elderly. In Workshop on Automation as Caregiver: the Role of Intelligent Technology in Elder Care (AAAI), August 2002.

[19] Aaron Powers and Sara Kiesler. The advisor robot: Tracing people's mental model from a robot's physical attributes. In Proceedings of the 1st ACM SIGCHI/SIGART Conference on Human-robot Interaction, HRI '06, pages 218-225, New York, NY, USA, 2006. ACM.

[20] Robin Read and Tony Belpaeme. Situational context directs how people affectively interpret robotic non-linguistic utterances. In Proceedings of the 2014 ACM/IEEE International Conference on Human-robot Interaction, HRI '14, pages 41-48, New York, NY, USA, 2014. ACM.

[21] P. Rouanet, J. Bechu, and P.-Y. Oudeyer. A comparison of three interfaces using handheld devices to intuitively drive and show objects to a social robot: the impact of underlying metaphors. In Robot and Human Interactive Communication, 2009. RO-MAN 2009. The 18th IEEE International Symposium on, pages 1066-1072, Sept 2009.

[22] Maha Salem, Micheline Ziadee, and Majd Sakr. Effects of politeness and interaction context on perception and experience of hri. In Guido Herrmann, MartinJ. Pearson, Alexander Lenz, Paul Bremner, Adam Spiers, and Ute Leonards, editors, Social Robotics, volume 8239 of Lecture Notes in Computer Science, pages 531-541. Springer International Publishing, 2013.

[23] Pericle Salvini, Cecilia Laschi, and Paolo Dario. Design for acceptability: Improving robots' coexistence in human society. International Journal of Social Robotics, 2(4):451-460, 2010.

[24] Karen A. Schriver. Dynamics in Document Design: Creating Text for Readers. John Wiley \& Sons, Inc., New York, NY, USA, 1997.

[25] Kerstin Severinson-Eklundh, Anders Green, and Helge Hüttenrauch. Social and collaborative aspects of interaction with a service robot. Robotics and Autonomous Systems, 42(3):223 - 234, 2003. Socially Interactive Robots.
[26] Megan Strait, Cody Canning, and Matthias Scheutz. Let me tell you! Investigating the effects of robot communication strategies in advice-giving situations based on robot appearance, interaction modality and distance. In Proceedings of the 2014 ACM/IEEE International Conference on Human-robot Interaction, HRI '14, pages 479-486, New York, NY, USA, 2014. ACM.

[27] Leila Takayama and C. Pantofaru. Influences on proxemic behaviors in human-robot interaction. In IEEE/RSJ International Conference on Intelligent Robots and Systems (IROS 2009), pages 5495-5502, October 2009.

[28] A Tapus, M.J. Mataric, and B. Scasselati. Socially assistive robotics [grand challenges of robotics]. Robotics Automation Magazine, IEEE, 14(1):35-42, March 2007.

[29] Benedict Tay, Younbo Jung, and Taezoon Park. When stereotypes meet robots: The double-edge sword of robot gender and personality in human-robot interaction. Computers in Human Behavior, 38:75-84, 2014.

[30] C. Torrey, S.R. Fussell, and S. Kiesler. How a robot should give advice. In 8th ACM/IEEE International Conference on Human-Robot Interaction (HRI 2013), pages 275-282, March 2013.

[31] J. Wainer, D.J. Feil-Seifer, D.A Shell, and M.J. Mataric. Embodiment and human-robot interaction: A task-based perspective. In Robot and Human interactive Communication, 2007. RO-MAN 2007. The 16th IEEE International Symposium on, pages 872-877, August 2007.

[32] JamesE. Young, Richard Hawkins, Ehud Sharlin, and Takeo Igarashi Toward acceptable domestic robots: Applying insights from social psychology. International Journal of Social Robotics, 1(1):95-108, 2009. 